Conclusion: The majority of activity limitations reported by patients receiving a structured OA care model in primary care were within the ICF Mobility domain. The most common third-level ICF domains were Changing body positions, Walking and Moving around. After participating in OA patient education and structured 8-12 weeks of exercise, the patients reported a statistically significant and clinically important improvement in the difficulty of performing their individual activities.

REFERENCES:

[1] Allen KD, Choong PF, Davis AM, et al. Osteoarthritis: Models for appropriate care across the disease continuum. Best practice \& research. Clinical rheumatology. 2016;30(3):503-535.

Disclosure of Interests: None declared

DOI: 10.1136/annrheumdis-2021-eular.2023

\section{POS0269-HPR CONTENT VALIDITY OF A MULTIPLE LANGUAGES QUESTIONNAIRE FOR MEASURING FLARE IN KNEE AND HIP OA: DEVELOPMENT OF THE FLARE-OA}

F. Guillemin ${ }^{1}$, A. Barcenilla-Wong ${ }^{2}$, M. Vitaloni ${ }^{3}$, L. Adarmouch ${ }^{4}$, M. T. Duruöz ${ }^{5}$, J. Epstein ${ }^{6}$, M. Sebbani ${ }^{4}$, Y. Traore ${ }^{6}$, C. Rutherford ${ }^{7}$, M. Cross $^{2}$, B. Fautrel ${ }^{8}$, T. Buttel $l^{9}$, G. A. Hawker ${ }^{10}$, L. March ${ }^{2}$, D. Hunter ${ }^{2}$, E. Spitz $^{1}$ on behalf of OMERACT Flares in OA. ${ }^{1}$ Université de lorraine, EA 4360 APEMAC, Metz, France; ${ }^{2}$ North Shore Private Hospital, Rheumatology, St Leonards, Australia; ${ }^{3} \mathrm{OAFI}$ Foundation (Osteoarthritis Foundation International), General, Barcelona, Spain; ${ }^{4}$ Université de Marrakech, Public Health, Marrakech, Morocco; ${ }^{5}$ Marmara University, Rheumatology, Istambul, Turkey; ${ }^{6}$ Université de Lorraine, School of Public Health, Nancy, France; ${ }^{7}$ University of Sydney, Faculty of Science, School of Psychology, Sydney, Australia; ${ }^{8}$ Assistance Publique - Hôpitaux de Paris, Rheumatology, Paris, France; ${ }^{9}$ OMERACT, Rheumatology, Sydney, Australia; ${ }^{10}$ University of Toronto - St. George Campus, Rheumatology, Toronto, Canada

Background: Flare in osteoarthritis $(\mathrm{OA})$ of the knee and hip $(\mathrm{KHOA})$ is an important outcome for patients' daily life and clinical research. A definition of a flare was set, and a core set of domains was recently endorsed by OMERACT/OARSI. No patient reported outcome (PRO) yet focusses on flare in OA specifically.

Objectives: To develop a self-reporting instrument measuring flare in 5 languages, using qualitative methods and international Delphi consensus.

Methods: We generated items using a dual-language (English and French) approach involving patients with OA from Australia, France and the United States and health care professionals (HCP) from international societies (OARSI, SFR, OMERACT). Item generation relied on semi-structured individual interviews conducted with OA patients and HCP and one focus group with patients. Content analysis allowed for identifying verbatim statements that were meaningful for patients and HCP. A Delphi consensus method was used to select the most relevant items, according to core domains set (OMERACT). A cross-cultural approach using current guidelines (1) was applied to produce Spanish (in Spain), Turkish and classical Arabic (in Morocco) versions using independent translation and expert committee to preserve its content validity.

Results: From semi-structured interviews with 29 patients and $16 \mathrm{HCPs}$ and one focus group with 10 patients, 180 statements in French (106) and English (77) were generated. Based on similarity or redundancy, 50 items with equivalent meaning in both languages were retained by an expert committee. After two Delphi rounds involving 50 patients and $116 \mathrm{HCPs}$ from 17 countries on four continents, it was reduced to 33 items (response $0=$ not at all, to $10=$ absolutely) in five domains (pain, swelling, stiffness, consequences of symptoms and psychological aspects). This questionnaire was cross-culturally adapted into Spanish, Turkish and classical Arabic. The Spanish version uncovered one inappropriate item in the original questionnaire that was amended accordingly in all 5 languages.

Conclusion: Flare is more than just an exacerbation of pain. The Flare-OA questionnaire includes all OMERACT recommended core domains. High content validity was seen in 2 original and 3 cross-culturally adapted languages. The Flare-OA should be considered as fit for purpose and evaluation of responsiveness in clinical studies in 5 languages.

Disclosure of Interests: None declared

DOI: 10.1136/annrheumdis-2021-eular.3870

\section{POS0270-HPR FLARE-OA QUESTIONNAIRE TO MEASURE FLARES IN OSTEOARTHRITIS OF THE KNEE AND HIP: ASSESSMENT OF ITS PSYCHOMETRIC PROPERTIES}

Y. Traore ${ }^{1}$, J. Epstein ${ }^{1,2}$, E. Spitz ${ }^{2}$, L. March ${ }^{3,4}$, J. F. Maillefert ${ }^{5}$,

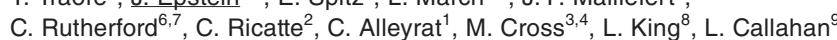
B. Fautrel ${ }^{10,11}$, T. Buttel ${ }^{3}$, G. A. Hawker ${ }^{8}$, D. Hunter ${ }^{3,4}$, F. Guillemin $1,2 \cdot{ }^{1} \mathrm{CHRU}$ Nancy, CIC 1433 EC, Nancy, France; ${ }^{2}$ Université de Lorraine, EA 4360 APEMAC, Nancy, France; ${ }^{3}$ University of Sydney, Institute of Bone and Joint Research, Kolling Institute, Sydney, Australia; ${ }^{4}$ Royal North Shore Hospital, Rheumatology Department, Sydney, Australia; ${ }^{5}$ Dijon University Hospital, Department of Rheumatology, Dijon, France; ${ }^{6}$ University of Sydney, Faculty of Science, School of Psychology, Sydney, Australia; ${ }^{7}$ University of Sydney, Faculty of Medicine and Health, Sydney Nursing School, Sydney, Australia; ${ }^{8}$ University of Toronto, Department of Medicine, Toronto, Canada; ${ }^{9}$ University of North Carolina, Thurston Arthritis Research Center, Chapel Hill, United States of America; ${ }^{10}$ Sorbonne Université - Assistance Publique Hôpitaux de Paris, Rheumatology Department Pitié-Salpêtrière Hospital, Paris, France; ${ }^{11}$ Institut Pierre Louis d'Epidémiologie et Santé Publique, INSERM UMR-S 1136, Paris, France

Background: Hip and knee OA is characterised by disease flares - understanding the determinants and consequences of $\mathrm{OA}$ flares has been hampered by lack of a standardized flare measure beyond the pain aspect. The patients point of view on the different aspect of their flares is essential.

Objectives: The objective was to assess the psychometric properties of a new questionnaire for measuring the multidimensional aspect of flares in $\mathrm{OA}$

Methods: Using a bilingual version of the questionnaire (33 items, response on a numeric scale, from 0 to 10), a multicentre survey (Australia, France and United States) was conducted on line with patients diagnosed with $O A$ of the hip and/or the knee. Based on an international OMERACT/OARS endorsed definition of the five core domains (pain, swelling, stiffness, consequences of symptoms and psychological aspects) composing a flare in OA [1], a confirmatory factorial analysis linked to the content analysis has been used to reduce the number of items and to determine the validity of the structure. The Flare-OA questionnaire (score from 0 to 100) has been tested in French and English for its internal consistency, its convergent validity with $\mathrm{HOOS} / \mathrm{KOOS}$ and Mini-OAKHQOL questionnaires, and its discriminant validity.

Results: Out of 398 patients (mean age 64 years old) who completed the questionnaire, $70.4 \%$ were female and $86.7 \%$ had knee OA. The confirmatory factorial analysis retained a model with 19 items (RMSEA $=0.06$; $\mathrm{SRMR}=0.04 ; \mathrm{CF}$ $=0.96$ and $\mathrm{TLI}=0.94$ ). The Cronbach Alpha was $>0.9$ for the 5 domains and for the whole questionnaire. The correlations between the Flare-OA and the other instruments were in line with that hypothesis flare is related but different from other concepts usually measured. The discriminant validity was evidenced by a significant score difference $(31.8 ; p<0,0001)$ between patients with and without flare, i.e. over twice the standard measurement error.

Conclusion: The optimized Flare-OA questionnaire (19 items) is a reliable and valid instrument freely available from the authors for measuring the frequency and severity of flare in knee and hip OA in clinical research.

REFERENCES:

[1] King LK, Epstein J, Cross M, et al. Establishing the Domains of Knee and Hip Osteoarthritis (OA) Flare: A Report from the OMERACT 2020 Inaugural Virtual Consensus Vote from the Flares in OA Working Group. (Submitted)

Disclosure of Interests: None declared

DOI: 10.1136/annrheumdis-2021-eular.3799 